\title{
Measurement Results of Transmit Delay Diversity for DVB-T Networks
}

\author{
R. Di Bari, M. Bard, J. Cosmas, R. Nilavalan, K.K. Loo, H. Shirazi, K. Krishnapillai \\ School of Engineering and Design, Brunel University \\ Uxbridge, Middlesex, UK \\ Raffaele.DiBari@brunel.ac.uk
}

\begin{abstract}
This paper describes work carried out by Brunel University and Broadreach Systems (UK) to quantify the advantages that can be achieved if Transmit Diversity is applied to systems employing the DVB standard. The techniques investigated can be applied to standard receiver equipment without modification. An extensive and carefully planned field trial performed during the winter of 2007/2008 in Uxbridge (UK) is described. The transmissions were performed in the $730 \mathrm{MHz}$ frequency band with a DVB-T transmitter and a mean power of $18.4 \mathrm{dBW}$. Transmit delay diversity has been observed to deliver significant reception improvement in automotive- non line of sight situations.
\end{abstract}

Keywords

Field trials, Transmit Delay Diversity, DVB-T.

\section{INTRODUCTION}

Multipath can result in severe fading in Non Line of Sight (NLOS) reception situations. A radio network design must take this into account when predicting service availability. Traditionally broadcast reception in multipath situations is provided by increasing the density of transmitters and increasing the radiated power. Transmit diversity is a technique where multiple transmitters, separated by only a few meters deliver signals to a NLOS receiver along radio channels with uncorrelated fading. The effect is to reduce the probability of observing deep fades at the receiver. A diversity gain will always be observed in NLOS situations provided the observed signals have uncorrelated fading and the receiver is able to exploit them, the result is that the number of transmitter sites and radiated power required to achieve effective coverage are both reduced. In this paper, an extensive and carefully planned field trial performed during the winter of 2007/2008 in Uxbridge (UK) is described. The transmissions were performed in the 730 $\mathrm{MHz}$ frequency band with a DVB-T transmitter and a mean power of $18.4 \mathrm{dBW}$. These trials were organized by Brunel University and Broadreach Systems.

\section{EXPERIMENTAL NETWORK}

The transmitter was located at Uxbridge's Brunel University, in the west outskirts of London, 1.2 Km south of Uxbridge. The measurement area is relatively flat and representative of a typical sub-urban area. Our field trial used two power amplifiers, rated at $100 \mathrm{~W}$, feeding an array of directive antennas.

In the tests we compared quality of reception in the measurement area when either all power was transmitted from a single antenna (Single In Single Out) or half power from each of 2 separate antennas (Multiple IN Multiple Out). The effective isotropic radiated power (EIRP) was 18.4 dBW for both the single transmitter and dual transmitter (MISO) configurations on channel 53 which has a central frequency of $730 \mathrm{MHz}$ and bandwidth of $8 \mathrm{MHz}$. The signals were compliant with the RF signal spectrum of DVB-T standard [2]. A directive antenna linear array was installed at the top of one of the university buildings (Tower D) pointing toward the east The antennas are horizontally polarized panels with a gain of $12 \mathrm{~dB}$, a halfpower beam-width of $53^{\circ}$ degrees in the $\mathrm{H}$ plane and $24^{\circ}$ degree in the $\mathrm{V}$ plane. A maximum number of two antennas (out of five) were active at any time. The spatial separations between active antennas could be selected from 8 to 27 wavelengths (Figure 1).

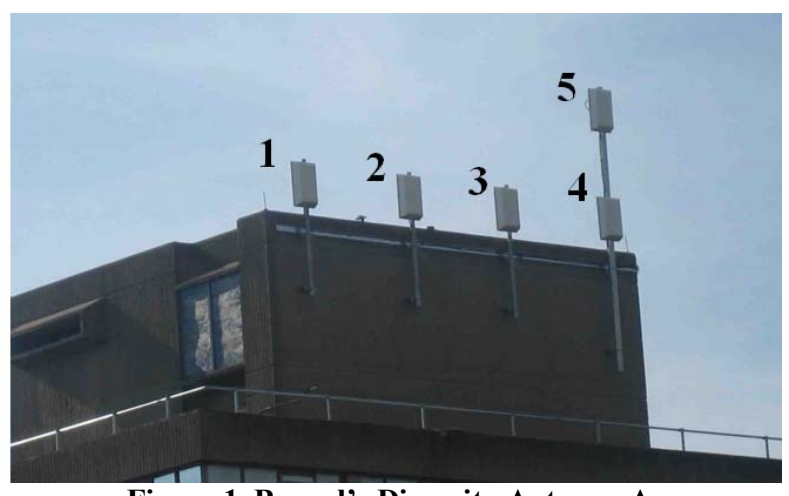

Figure 1. Brunel's Diversity Antenna Array

The total feeder loss was $6.8 \mathrm{~dB}$ and the switch matrix connecting the amplifier $\mathrm{B}$ with one of the diversity antennas had a loss of $0.2 \mathrm{~dB}$. In Table 1 the main technical characteristics of the transmission are summarized.

Pseudo Random Binary Sequence (PRBS) packets were used for field measurements. The system is also capable of delivering multimedia content, encoded into MPEG-2 Transport Streams (TS) packets and transmitting it over the network for demonstration purposes. A standard DVB-T Modulator was used to feed IF signals directly into the Broadreach Diversity Unit (custom made), introducing a 
$1.1 \mu s$ relative delay between the two signals at the output.

Fig. 2 shows the schematic of the transmit system.

Table 1. Technical characteristics of the transmission

\begin{tabular}{|l|l|}
\hline Transmitter Centre & Uxbridge \\
\hline Broadcaster & Brunel University \\
\hline Coordinates & $51.532367 \mathrm{~N} ; 0.473218 \mathrm{~W}$ \\
\hline Frequency & $730 \mathrm{MHz}$ \\
\hline Bandwidth & $8 \mathrm{MHz}$ \\
\hline EIRP & $18.4 \mathrm{dBW}$ \\
\hline Antenna & Directive/Horizontal Polarized \\
\hline Antenna(s) gain & $12 \mathrm{~dB}$ \\
\hline Antennas separations & $8,16,24 \lambda$ horizontal or \\
& $24 \lambda$ horizontal and $7 \lambda$ vertical. \\
\hline Total feeders loss & $6.8 \mathrm{~dB}(6.6 \mathrm{~dB}+0.2 \mathrm{~dB})$ \\
\hline $\begin{array}{l}\text { Height of the antenna above } \\
\text { the ground }\end{array}$ & $25 \mathrm{~m}$ \\
\hline
\end{tabular}

The mobile reception was measured using a car equipped with a DVB measurement system provided by Broadreach Systems. Fig. 3 illustrate the integrated system used to record seconds in which uncorrectable Reed-Solomon blocks are observed, from this the Errored Seconds Ratio (ESR) is calculated, this is the ratio of errored to nonerrored seconds. The Signal to Noise Ratio (SNR) and the received signal strength indication (RSSI) are also measured together with location coordinates acquired using a global position system (GPS) receiver. The Broadreach monitor receiver comprised a DVB-T demodulator measuring the signal quality, an integral processor to store data and provide user interfaces, and a GPS receiver. The signal was received using an omnidirectional horizontally polarized antenna, installed on the rooftop of the test vehicle. This system was used to measure the ESR, SNR, and RSSI, every measurement was recorded and tagged with the corresponding location coordinates.

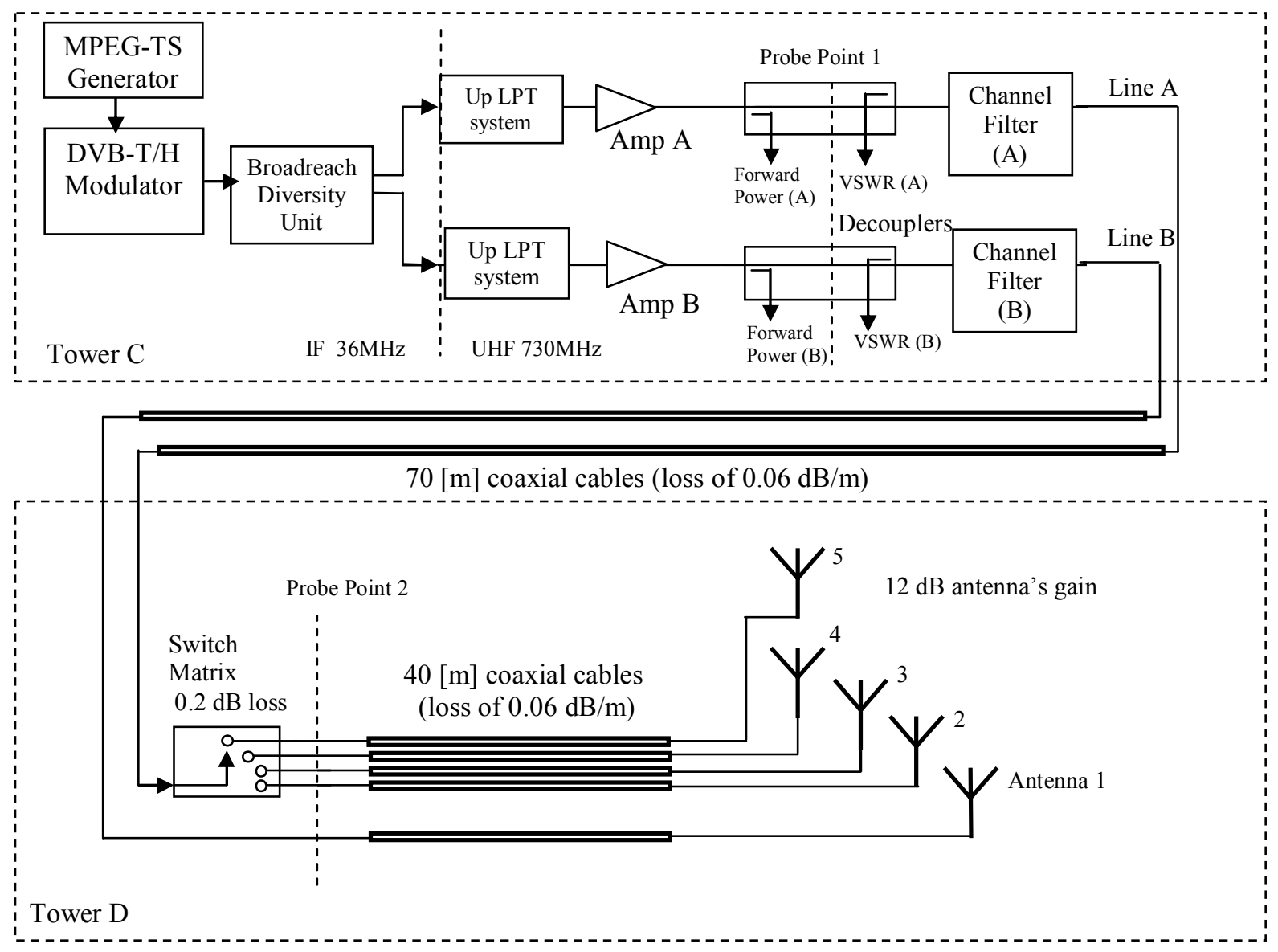

Figure 2. The RF diversity transmit system 


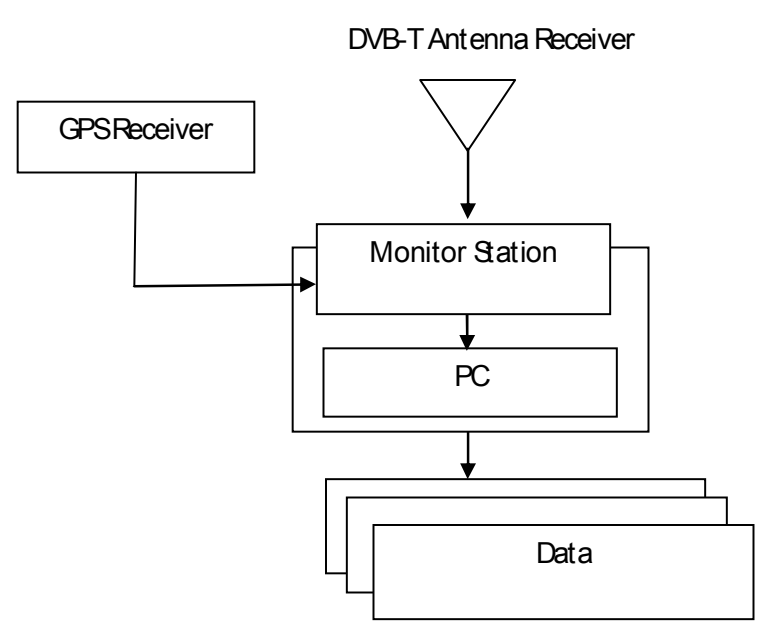

Figure 3. The car experimental set-up

\section{MEASUREMENT METHODOLOGY}

\section{Measurement metrics}

A complete set of radio channel and signal data were gathered every second. The parameters of interest include the Errored Seconds (ES), the RSSI, and the SNR. The ES denotes the number of seconds containing faulty MPEG-TS packets. Errored seconds are a good metric as they are observable in the received picture and therefore reflect the impact of signal quality on the subscriber's experience. A total errored second ratio (ESRT) per each experiment is defined as:

$\operatorname{ESRT}[\%]=\frac{E S}{\text { total number of measurement } \sec \text { onds }} \times 100$.

The ESRT gain relative to SISO is defined as

$G_{E S R T, S I S O}[\%]=\frac{\operatorname{GAIN}_{\text {ESRT }}}{E S R T_{\text {SISO }}} \times 100$,

where $G_{E S R T}[\%]=E S R T_{S I S O}-E S R T_{M I S O}$. Reference [3] defines the ESR5 metric (erroneous seconds ratio at 5\%) to denote a maximum of one erroneous second in 20 seconds as the maximum rate for acceptable reception. Thus the percentage of good reception $R$ during each experiment is defined as

$R[\%]=\frac{\text { total number of ESR } 5 \text { clusters }}{\text { total number of } 20 \mathrm{sec} \text { onds clusters }} \times 100$.

The reception gain $G_{R}$ is defined as

$$
G_{R, S I S O}[\%]=\frac{G_{R}}{R_{\text {SISO }}} \times 100,
$$

where $G_{R}[\%]=R_{\text {SISO }}-R_{\text {MISO }}$. The RSSI and the SNR are both estimated at the beginning of each measurement second.

\section{Area classification and route selection}

The area in which the measurements were conducted was classified into six different environments (Figure 3 and Figure 4). The characteristics of those environments are:

- "University Campus" (A Zone) includes high buildings, offices, and residential halls. The typical height of these buildings are $30 \mathrm{~m}$ and the area includes several trees. Several locations are in LOS with the transmitter antenna array.

- "Sub-urban" (B Zone) can be described as a low density sub-urban area, with a significant presence of trees and vegetation. Residential streets within this zone are typically 10 meters wide.

- "Playground fields" (C zone) include typically football or tennis playgrounds, without a significative presence of buildings or trees.

- "Trees areas" (D zone) is of the same type as the playground fields' zone, but with a significative presence of trees.

- "Wide roads" (E zone) are 19.7 meters wide roads, 2 lanes each way, holding moderate traffic and surrounded by 2 floor residential houses. The speeds on these roads varied significantly with many accelerations and decelerations due to the variable traffic level,

- "Medium roads" (F zone) are 11.5 meters wide roads with 1 lane each way. They hold light traffic, but the speeds varied significantly.

The two selected routes covered mainly sub-urban zones and included wide and medium roads in all directions reaching marginal areas of Brunel's service coverage (Route B). Route A was centered around Brunel's transmit site, approximately $7.7 \mathrm{Km}$ long, and was selected mainly to investigate the antenna performance. Route B was in the main direction of the transmit power, it is approximately 13.2 $\mathrm{Km}$ long and was selected mainly to measure the diversity gain. The maximum range covered by Route B was $1.72 \mathrm{Km}$. It should be mentioned that the measurements were repeated two or three times on the same routes. In total more than $447 \mathrm{~km}$ of mobile measurements were recorded. Fig. 4 and 5 show the two selected routes. Typical speeds were in the range of 0 to $48.2 \mathrm{Km} / \mathrm{h}$, with an average of $30 \mathrm{Km} / \mathrm{h}$. The measurements taken when the receiver was stationary were removed from the data presented here to eliminate the effects of random stops due to traffic lights etc on the total reception statistics. 


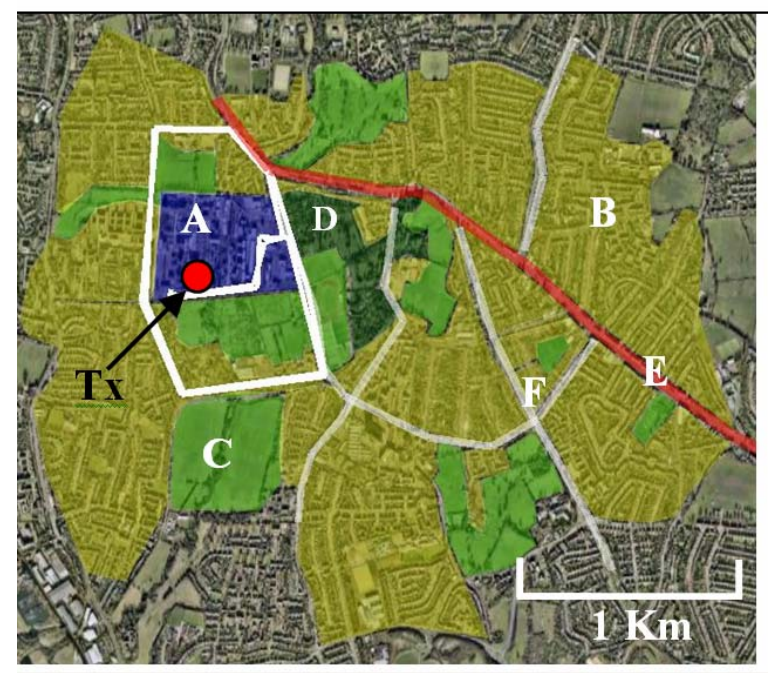

Figure 4. Route $A$ and areas classification

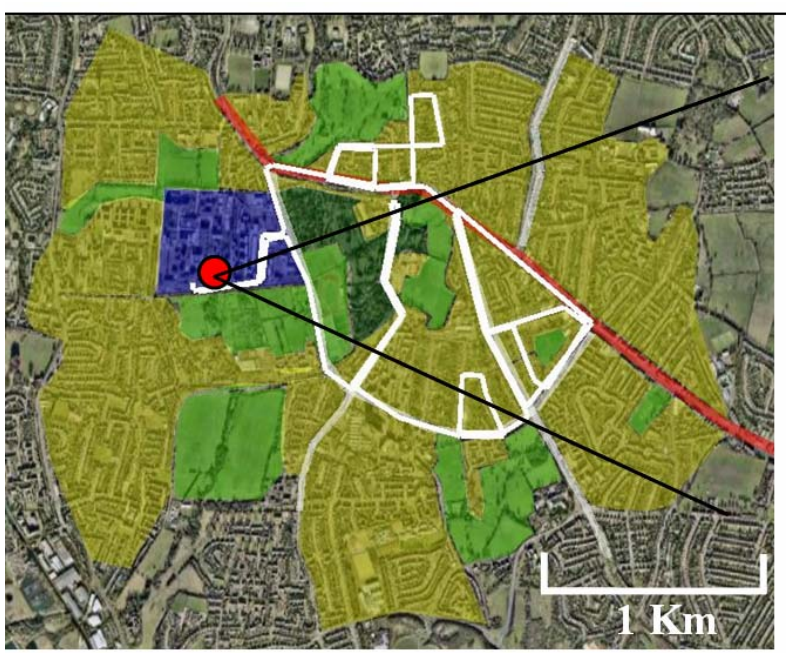

Figure 5. Route B and transmit antenna's 3dB beam-width

\section{Verification of the transmitter set-up}

The signal quality was tested at several stages in the transmit chain to ensure it was acceptable. The relative levels of the spectrum shoulders are fully compliant with the RF signal spectrum mask of DVB-T standard [2]. The two amplifiers did not introduce any significant non-linear effects when operating on SISO configuration (e.g. at full power). The power transfer system was calibrated and the power levels were carefully measured, adjusted and monitored during the measurements using a power measurement device with calibration traceable to national standards. In the MISO case each antenna transmitted half of the EIRP of the SISO case. A preliminary set of driving measurements was performed on the radial route A to test the average RSSI and SNR. Since the antennas transmit the same EIRP for the SISO and the MISO cases, it was expected that the average RSSI and SNR parameters will be approximately equal for all configurations around this radial route. Table 2 shows the measured RSSI and SNR values and it can be seen that they do not differ significantly from the SISO antenna 1 to the SISO antenna 4 cases.

Table 2. Results for probing the offsets of the ESRT, the average RSSI and SNR along the route $A$

\begin{tabular}{|l|l|l|l|}
\hline & $\begin{array}{l}\text { ESRT }_{\text {SISO1- }^{-}} \\
\text {ESRT }_{\text {SISO4 }^{-}}\end{array}$ & $\begin{array}{l}\text { RSSI }_{\text {IISO }^{-}} \\
\text {RSSI }_{\text {SISO4 }}(\mathrm{dB})\end{array}$ & $\begin{array}{l}\text { SNR }_{\text {SISO1- }} \\
\text { SNR }_{\text {SISO4 }} \\
(\mathrm{dB})\end{array}$ \\
\hline DAY 1 & $1 \%$ & 0.7 & -0.3 \\
\hline DAY 2 & $-1.1 \%$ & 1 & -0.1 \\
\hline
\end{tabular}

\section{MOBILE RECEPTION RESULTS}

Further measurements were performed along route B to investigate mobile reception. The time required for driving along this route was approximately 30 minutes. The diversity gain was estimated by comparing SISO and MISO driving tests performed sequentially to ensure analogous traffic conditions for the two measurements. Weather conditions were sunny or partly cloudy and background traffic was generally light/medium. The tests results presented here were obtained employing the broadcast conditions described in Table 3.

Table 3. DVB-T system parameters

\begin{tabular}{|l|l|l|}
\hline Configuration Name & SISO & MISO \\
\hline Code Rate & $3 / 4$ \\
\hline Signal Constellation & 4-QAM \\
\hline DVB-T Mode & $2 \mathrm{k}$ \\
\hline Hierarchy $(\alpha)$ & Non hierarchical \\
\hline Guard Interval & $1 / 32$ & \multicolumn{2}{|l|}{} \\
\hline Bitrate (Mbit/s) & 9.05 & $\begin{array}{l}15.4 \text { (ant. 1) }+15.4 \\
\text { ant. 4) }\end{array}$ \\
\hline EIRP (dBW) & 18.4 & 24 \\
\hline Antenna separation $(\lambda)$ & - & 1.1 \\
\hline Diversity Delay $(\mu s)$ & - &
\end{tabular}

\section{Diversity across several days}

Figure 6 shows the ESRT for SISO and MISO (left axis) and their relative $G_{E S R T}$ and $G_{E S R T, S I S O}$ (right axis). The measurements were taken during different days, and arranged with $G_{E S R T}$ descending. The MISO performances were compared with the SISO performances for antenna 1. A $90 \%$ confidence interval is derived from Student's tdistribution. The sample mean $G_{E S R T}$ is about $14.9 \%$ with a sample variance of $4.9 \%$ and a $90 \%$ confidence interval lower and upper bound equal to $7.2 \%$ and $22.6 \%$, respectively. The $G_{E S R T}$ values out of this interval (e.g. the first, the second and the last $G_{E S R T}$ point on the chart) are probably due to significantly different traffic conditions for the measurements compared. The sample mean $G_{E S R T, S I S O}$ is about $31.1 \%$ with a sample variance of $11.3 \%$ and a $90 \%$ 
confidence interval lower and upper bound equal to $19.4 \%$ and $42.8 \%$, respectively.

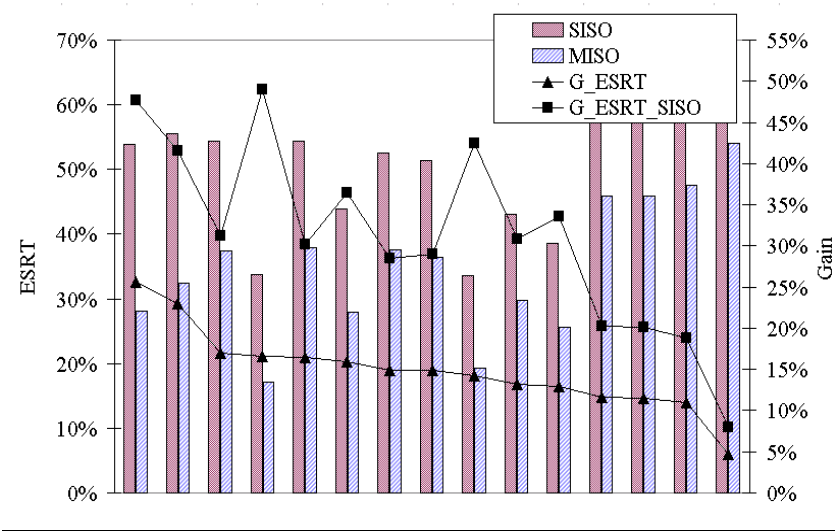

Figure 6. ESRT of all experiments (left axis) with $G_{E S R T}$ and $G_{E S R T, S I S O}$ (right axis).

Figure 7 shows the percentage of good Reception (where ESR is equal or less than 5\%) for SISO and MISO (left axis) and their relative $G_{E S R T}$ and $G_{E S R T, S I S O}$ (right axis). The measurements were taken during different days, and arranged with $G_{R}$ descending. The sample mean $G_{R}$ is about $14.3 \%$ with a sample variance of $7.4 \%$ and a $90 \%$ confidence interval lower and upper bound equal to $4.8 \%$ and $23.8 \%$, respectively. The sample mean $G_{R, S I S O}$ is about $22.2 \%$ with a sample variation of $12.4 \%$ and a $90 \%$ confidence interval lower and upper bound equal to $9.9 \%$ and $34.4 \%$, respectively. In Table 4 the means, variances and $90 \%$ confidence interval are summarized.

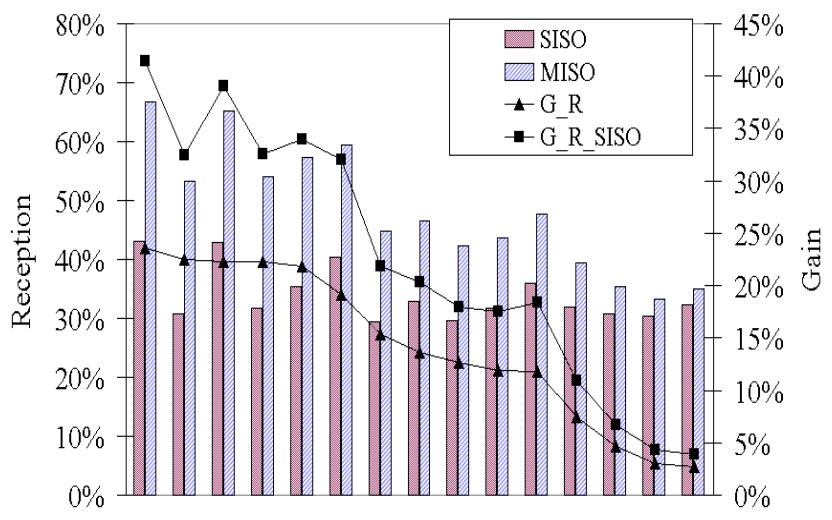

Figure 7. Reception of all experiments (left axis) with $G_{R, S I S O}$ and $G_{R}$ (right axis).

As described in the previous section, $G_{E S R T, S I S O}$ and $G_{R, S I S O}$ consist of the $G_{E S R T, S I S O}$ and $G_{R, S I S O}$ normalized by the SISO performance (e.g. $E S R T_{S I S O}$ and $R_{S I S O}$ ).

The data high variances are probably due to different the traffic conditions for the measurements compared.
However, the results clearly show that the MISO performances were always better than SISO.

Table 4. Sample mean and variance of the gain measurements and confidence intervals derived from Student's t-istribution.

\begin{tabular}{|c|c|c|c|c|}
\hline & \multirow{2}{*}{$\begin{array}{l}\text { sample } \\
\text { mean } \\
{[\%]}\end{array}$} & \multirow{2}{*}{$\begin{array}{l}\text { sample } \\
\text { variance } \\
{[\%]}\end{array}$} & \multicolumn{2}{|c|}{$90 \%$ confidence intervals } \\
\hline & & & $\begin{array}{l}\text { lower } \\
\text { bound [\%] }\end{array}$ & $\begin{array}{l}\text { upper } \\
\text { bound [\%] }\end{array}$ \\
\hline$G_{E S R T}$ & 14.9 & 4.9 & 7.2 & 22.6 \\
\hline$G_{E S R T, S I S O}$ & 31.1 & 11.3 & 19.4 & 42.8 \\
\hline$G_{R}$ & 14.3 & 7.4 & 4.8 & 23.8 \\
\hline$G_{R, S I S O}$ & 22.2 & 12.4 & 9.9 & 34.4 \\
\hline
\end{tabular}

Qualitative analysis of a sample pair experiment results This section shows the measurement metrics of a sample pair of SISO and MISO measurements plotted versus the corresponding geographical coordinates. Figure 8 and Figure 9 show the distribution of the SNR and the RSSI along Route B. Although the two figures are for SISO measurements they can be viewed as a general indicator for the MISO measurement as well. The squares in the picture (named as S1,S2, S3, and S4) indicate route sections of interest for subsequent analysis. This route selected covers a wide range of SNR and RSSI levels, summarized in the Table 5.

Table 5. SNR and RSSI level ranges classification

\begin{tabular}{|l|l|l|l|}
\hline & $\begin{array}{l}\text { High } \\
\text { Range }\end{array}$ & $\begin{array}{l}\text { Medium } \\
\text { Range }\end{array}$ & $\begin{array}{l}\text { Low } \\
\text { Range }\end{array}$ \\
\hline SNR (dB) & $24-17.5$ & $17.5-11$ & $11-4.5$ \\
\hline $\begin{array}{l}\text { RSSI } \\
(\mathrm{dBm})\end{array}$ & -48.52 to - & -63.6 to - & -73.3 to - \\
\hline
\end{tabular}

Figure 10 and Figure 11 show the errored and errors free seconds occurrences along the route for SISO and MISO reception. Sections S1, S2, and S3 indicate sectors where a significant reduction in ES was observed.

Figure 12 shows where SISO reception is acceptable (ESR $\leq 5 \%$ ) along $42.8 \%$ of the route. Reception was not possible at the points furthest from the transmitter where the SNR and RSSI values were at their lowest levels.

Figure 13 shows the impact of diversity on reception. Diversity enables reception within sectors S1, S2, and S4 where the RSSI is mostly in the medium range level and SNR varies between the low and medium levels, reception is now possible for $65.1 \%$ of the route. It can be noted that enhancement in diversity reception is observed mostly in sections S1 and S2, corresponding to sub-urban, wide and medium road zones. In those zones, the SNR and RSSI levels may be sufficiently high to enable stationary SISO reception, but because of the receiver speed, reception is possible only if diversity is applied. 


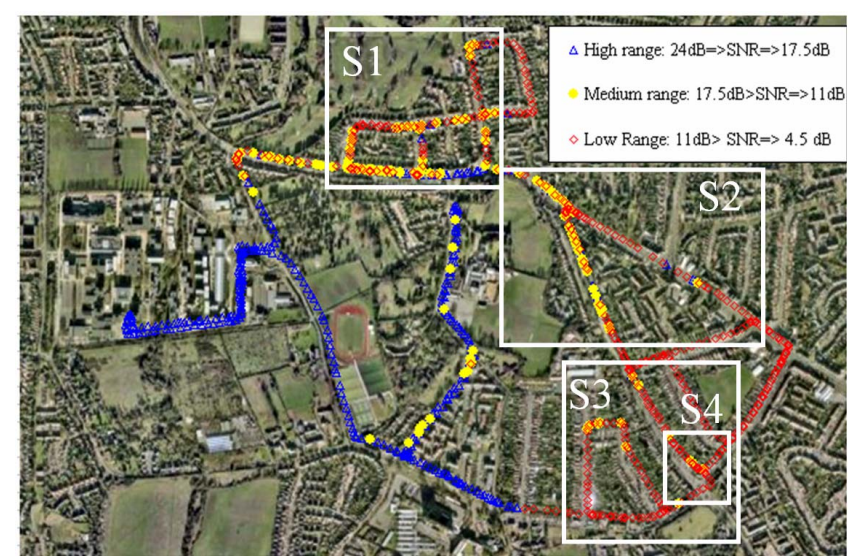

Figure 8. SNR profile along the Route B for SISO configuration.

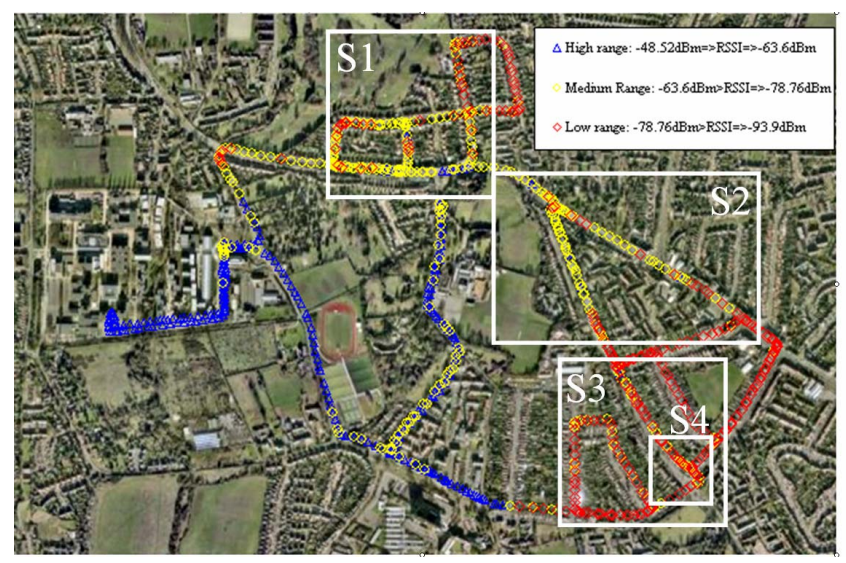

Figure 9. RSSI profile along the Route B for SISO configuration.

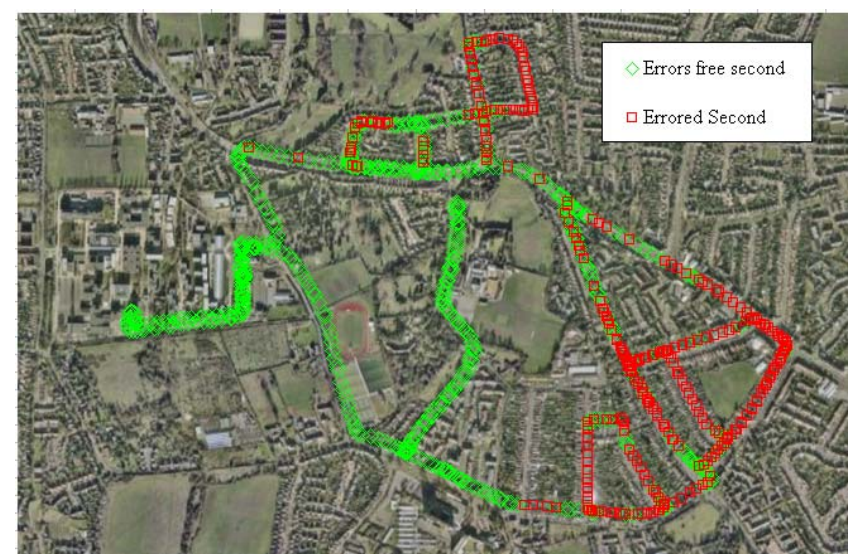

Figure 10. Errored and errors free seconds for SISO reception along Route B

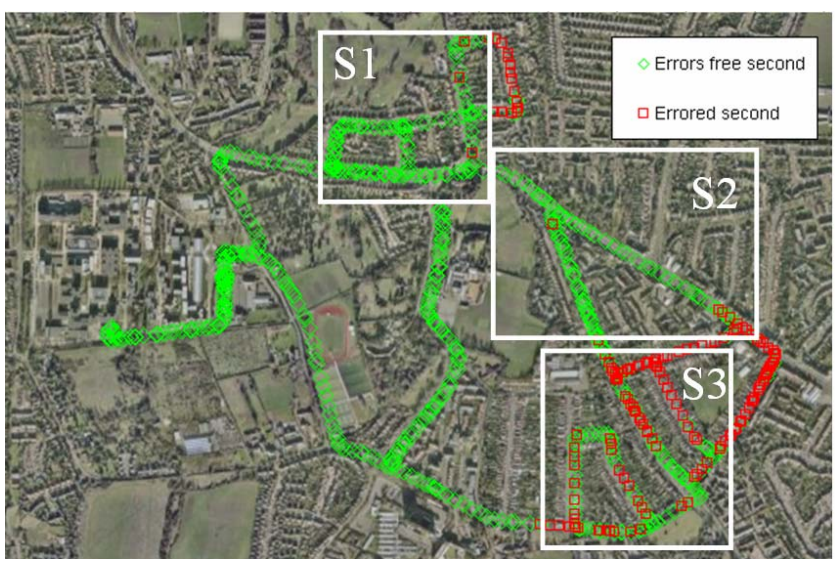

Figure 11. Errored and errors free seconds for MISO reception along Route $B$. The squares indicate the route sectors where a reduction of ES was observed.

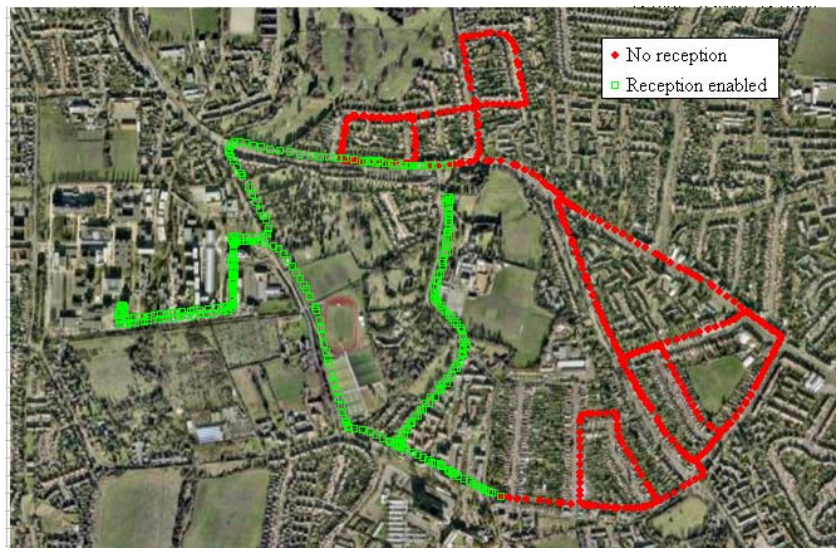

Figure 12. SISO reception along Route B

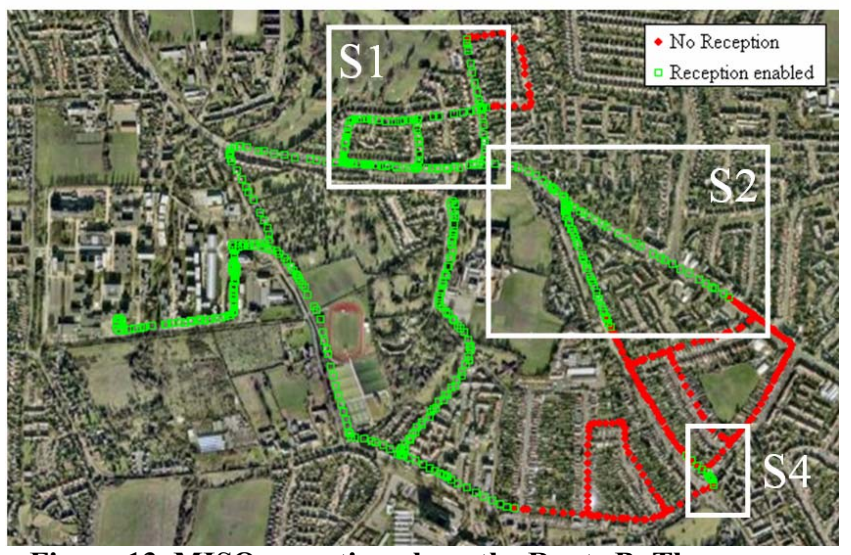

Figure 13. MISO reception along the Route $B$. The squares indicate the route sectors where a major reception gain was observed. 


\section{CONCLUSIONS}

This paper presents experiments and DVB-T test results investigating the effectiveness of a transmit diversity system. The field trial has been designed to validate simulation and laboratory results and to provide realisable performance figures.

In sub-urban areas, the number of errored seconds has been reduced and the instances of acceptable reception have been significantly increased. The relative $90 \%$ confidence intervals have also been presented. Finally, the SNR and the RSSI ranges where diversity gain occurs have been estimated by a qualitative analysis. Future works will consist of a generalised analysis of reception and coverage enhancement an extended by conducting a measurement campaign indoors. The tests and results presented are for enhancement techniques that can be applied to Broadcasting system utilising existing DVB standards without modification. The improvements can be exploited at the network level since there is no impact on receiver design. Resulting DVB broadcast networks could have fewer transmitter sites or lower EIRP due to the increased coverage delivered by these techniques, and thus would be more cost-effective and have less environmental impact.

\section{ACKNOWLEDGMENTS}

The authors would like to thank Antonio Petrolino from INESC-ID (PO) and Pierpaolo Vivo from Brunel University (UK) for their helpful comments.

\section{REFERENCES}

[1] Y. Zhang, J. Cosmas, K.-K. Loo, M. Bard, and R. D. Bari, "Analysis of Cyclic Delay Diversity on DVB-H Systems over Spatially Correlated Channel", IEEE Transactions on Broadcasting, Vol.53, No.1, March 2007.

[2] R. Di Bari, M. Bard, Y. Zhang, K.M. Nasr, J. Cosmas, K.K. Loo, R. Nilavalan, H. Shirazi, K. Krishnapillai, "Laboratory Measurement Campaign of DVB-T Signal with Transmit Delay Diversity", to appear in IEEE Transactions on Broadcasting Special Issue on "Quality Issue in Multimedia Broadcasting", 2008.

[3] European Telecommunications Standard Institute ETSI, Digital Video Broadcasting (DVB); Framing structure, channel coding and modulation for digital terrestrial television, July 1999, EN 300744 V1.2.1.

[4] The ESR5 criterion for the Assessment of DVB-T transmission Quality, ITU-R Document 6E/64-E, April 8, 2004. 